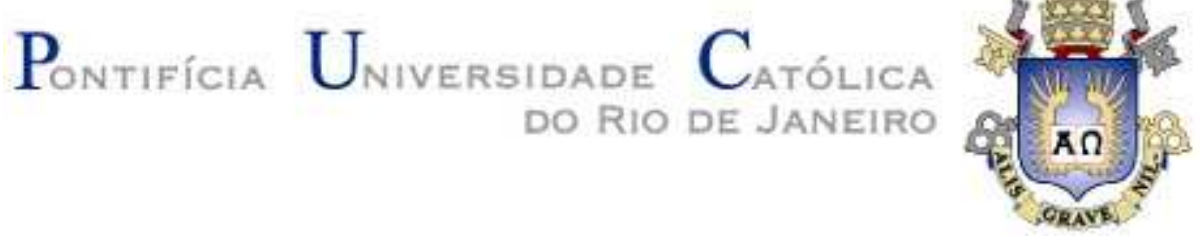

Eduardo da Cunha Moreira

Gestão de Custos Logísticos baseada em atividades e no custo total de propriedade

Dissertação de Mestrado

Dissertação apresentada como requisito parcial para obtenção do título de Mestre pelo Programa de PósGraduação em Engenharia Industrial da PUC-Rio.

Orientador: Prof. Paulo Henrique Soto

Rio de Janeiro

Março de 2008 


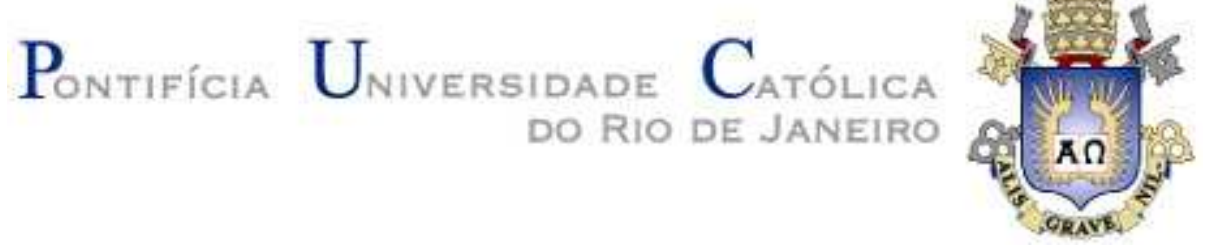

Eduardo da Cunha Moreira

\section{Gestão de Custos Logísticos baseada em atividades e no custo total de propriedade.}

Dissertação apresentada como requisito parcial para obtenção do título de Mestre pelo Programa de Pós-Graduação em Engenharia Industrial da PUC-Rio. Aprovada pela Comissão Examinadora abaixo assinada.

Paulo Henrique Soto

Orientador

PUC-Rio

Paulo Henrique Soto PUC-Rio

Nélio Domingues Pizzolato

PUC-Rio

Carlos Patrício Samanez

PUC-Rio

José Eugênio Leal

Coordenador(a) Setorial do Centro Técnico Científico - PUC-Rio 
Todos os direitos reservados. É proibida a reprodução total ou parcial do trabalho sem autorização da universidade, do autor e do orientador.

\section{Eduardo da Cunha Moreira}

Graduou-se em Engenharia de Produção Mecânica na Universidade do Estado do Rio de Janeiro em 2002. Estagiou na área de Engenharia de Manufatura da Xerox do Brasil e na área de Qualidade Assegurada da Volkswagen do Brasil . Após a graduação trabalhou na FMC CBV Subsea, onde atuou na área de planejamento da produção. Atualmente é funcionário da Petróleo Brasileiro S.A. e trabalha na área de Logística na Diretoria do Abastecimento.

Ficha Catalográfica

Moreira, Eduardo da Cunha

Gestão de custos logísticos baseada em atividades e no custo total de propriedade / Eduardo da Cunha Moreira ; orientador: Paulo Henrique Soto. $-2008$.

63 f. : il. ; $30 \mathrm{~cm}$

Dissertação (Mestrado em Engenharia Industrial)-Pontifícia Universidade Católica do Rio de Janeiro, Rio de Janeiro, 2008.

Inclui bibliografia

1. Engenharia industrial - Teses. 2. Custo logístico. 3. Custeio baseado em atividade. 4. Custo total de propriedade. I. Soto, Paulo Henrique. II. Pontifícia Universidade Católica do Rio de Janeiro. Departamento de Engenharia Industrial. III. Título. 


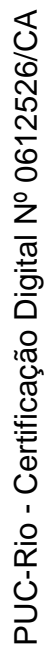

Para minha Família. 


\section{Agradecimentos}

A minha mãe Marina e minha irmã Renata pelo incentivo, mesmo que à distância.

A minha namorada Paula Valente pela compreensão com minha limitação de tempo livre.

Aos meus amigos pela compreensão nos momentos de ausência.

Ao meu gerente geral Carlos Felipe Lodi e ao meu gerente Abdias Fernandes pela indicação ao curso, apoio e incentivo.

Aos colegas de trabalho da Petrobras pelo apoio e informações indispensáveis para realizar o trabalho.

A Petróleo Brasileiro S.A pela oportunidade de realizar o curso com todo o apoio necessário.

Ao Prof. Paulo Henrique Soto Costa pelo suporte e orientação. 


\section{Resumo}

Moreira, Eduardo da Cunha; Soto, Paulo Henrique. Gestão de Custos Logísticos baseada em atividades e no custo total de propriedade. Rio de Janeiro, 2008. 63p. Dissertação de Mestrado - Departamento de Engenharia Industrial, Pontifícia Universidade Católica do Rio de Janeiro.

A correta avaliação dos custos logísticos de uma empresa permite uma melhor gestão destes custos e tomada de decisões mais precisas. Este trabalho aborda a apuração de custos logísticos através do método de custeio tradicional, indica os problemas que o tornam inadequado para esta tarefa e propõe a utilização de ferramentas contemporâneas. Apesar de considerar a importância da análise dos custos logísticos totais, o trabalho desenvolve a análise somente para os custos do transporte marítimo, pois estes são os mais relevantes para o caso em análise. Para solucionar as inadequações do método tradicional de custeio, é proposta a utilização do método de Custeio Baseado em Atividades, do inglês Activity-Based Costing ( $\mathrm{ABC}$ ). Este método visa atribuir os custos dos recursos de acordo com as atividades onde estes foram utilizados e traz bons resultados na avaliação gerencial dos custos logísticos. Além deste método, é proposta a utilização do Custo Total de Propriedade, do inglês Total Cost of Ownership (TCO), para a contratação de navios. Esta ferramenta avalia o custo de obtenção e uso de um bem ou serviço, incluindo outras variáveis além do preço. Os resultados do trabalho são favoráveis à utilização das ferramentas, mas há necessidade de um extenso trabalho interno no seu ambiente de aplicação para a sua implementação.

\section{Palavras-chave}

Custo Logístico; Custeio Baseado em Atividade; Custo Total de Propriedade. 


\section{Abstract}

Moreira, Eduardo da Cunha; Soto, Paulo Henrique. Logistical c o s t s management based on activities and total cost of ownership. Rio de Janeiro, 2008. 63 p. M. Sc. Dissertation - Departamento de Engenharia Industrial, Pontifícia Universidade Católica do Rio de Janeiro.

The correct evaluation of the logistical costs of a company allows better management of these costs and a more accurate decision making process. This work addresses the obtainment of logistical costs through the traditional method of cost assignment, points out problems that make it unsuitable for this task, and suggests the use of contemporary tools. Despite considering the importance of the total logistical costs, this work develops the analysis only for the costs of shipping, since these are the most relevant to the case under approach. To solve the inadequacies of the traditional method of cost assignment, it is suggested the Activity-Based Costing method (ABC). This method allocates the cost of resources in accordance with the activities over which they were used and brings forth good results in the managerial evaluation of logistical costs. In addition to this method, it is suggested the use of Total Cost of Ownership (TCO) for hiring ships. This tool evaluates the cost of obtaining and using a good or service, including other variables besides price. The results of this work are favourable to the use of the tools, but there is the need of extensive internal work in its field of application to have it implemented.

\section{Keywords}

Logistic Cost; Activity-Based Cost; Total Cost os Ownership 


\section{Sumário}

1 Introdução 12

1.1. Motivação 15

1.2. Objetivo 15

1.3. Delimitação de Escopo 16

1.4. Metodologia de pesquisa 16

$\begin{array}{ll}\text { 1.5. Estrutura do trabalho } & 16\end{array}$

2 O Custeio Tradicional 13

3 Custos Logísticos 19

3.1. Logística de abastecimento e custos 22

3.2. Logística da planta e custos 23

3.3. Logística de distribuição e custos 23

4 Custeio e Avaliação de Desempenho 22

4.1. Custos do Transporte Marítimo 25

4.1.1. Composição dos custos de transporte marítimo 28

5 Ferramentas Contemporâneas de Custeio 25

5.1. Custeio Baseado em Atividades - ABC 25

5.2. Aplicação do $A B C$ na avaliação do custo do Transporte Marítimo 34

5.2.1. Análise das informações de custos 39

5.3. Custo total de propriedade (Total cost of ownership - TCO) 40

5.3.1. Proposta de utilização do TCO na área de Contratação de Navios 42

5.3.2. Benefícios da utilização do TCO 44

5.3.3. Dificuldade na implementação do TCO 46

5.3.4. Modelos de identificação de custos do TCO 46

5.4. Modelo para avaliação de custo total de propriedades para contratos 
de navios tipo Time Party Charter (TCP)

6 Considerações finais e recomendações futuras

6.1. Considerações Finais

6.2. Recomendações Futuras

7 Referências Bibliográficas 


\section{Lista de figuras}

Figura 1 Custos Logísticos como percentual do valor agregado. 14

Figura 2 Custos Logísticos no Brasil em 2004

Figura 3 Composição percentual média da frota por tipo

de contrato 2006

Figura 4 Percentual por tipo de navegação em 2006.

Figura 5 Percentual da quantidade em tonelada por tipo de produto transportado por Cabotagem em 2006

Figura 6 Custo do Transporte Marítimo x Carga Transportada 2003 até 2006

Figura 7 Modelo básico de ABC 30

Figura 8 Gerenciamento baseado em atividade - operacional e estratégico 34

Figura 9 Gráfico de despesas por tipo de contrato e atividade $\quad 39$

Figura 10 Despesas com distribuição por contrato e mercado 40

Figura 11 Composição do custo de transporte marítimo em 2006.

Figura 12 Custos totais de uma rede de distribuição 52

Figura 13 Modelo de Custo total/ Valor 54 


\section{Lista de tabelas}

Tabela 1 Custos médio das empresas 2005,2006 e $2007 \quad 19$

Tabela 2 Grupos de contas contábeis 28

Tabela 3 Análise estratégica de custos e o ABC 31

Tabela 4 Participação percentual no total dos custos por tipo de navio. 32

Tabela 5 Tipos de Navios 33

Tabela 6 Gastos com Navios em 2007

Tabela 7 Valores dos direcionadores de recursos 36

Tabela 8 Custos de sobrestadia $\quad 36$

Tabela 9 Despesas alocadas por atividades 37

Tabela 10 Valores dos direcionadores de atividades 38

Tabela 11 Despesas alocadas por objetos 38

Tabela 12 Pontos perdidos por evento. 49

Tabela 13 Nota média dos armadores $\quad 49$

Tabela 14 Preços de navios por armador $\quad 50$

Tabela 15 Custo de entrega $\quad 50$

Tabela 16 Custo de devolução $\quad 50$

Tabela 17 Valores dos componentes de cálculo do IDF 51

Tabela 18 IDF e Custo Total 51 